\title{
Editorial
}

\section{Smart Spectrum Technologies for Mobile Information Systems}

\author{
Miguel López-Benítez, ${ }^{1}$ Janne Lehtomäki, ${ }^{2}$ Kenta Umebayashi, ${ }^{3}$ and Fernando Casadevall ${ }^{4}$ \\ ${ }^{1}$ Department of Electrical Engineering and Electronics, University of Liverpool, Liverpool L69 3GJ, UK \\ ${ }^{2}$ Centre for Wireless Communications, University of Oulu, 90014 Oulu, Finland \\ ${ }^{3}$ Department of Electrical and Electronic Engineering, Tokyo University of Agriculture and Technology, Fuchu 184-8588, Japan \\ ${ }^{4}$ Department of Signal Theory and Communications, Technical University of Catalonia, 08034 Barcelona, Spain
}

Correspondence should be addressed to Miguel López-Benítez; m.lopez-benitez@liverpool.ac.uk

Received 28 July 2016; Accepted 31 July 2016

Copyright (C) 2016 Miguel López-Benítez et al. This is an open access article distributed under the Creative Commons Attribution License, which permits unrestricted use, distribution, and reproduction in any medium, provided the original work is properly cited.

Despite being one of the most important resources of mobile information systems, the radio frequency spectrum has usually been sparsely exploited as a result of the static spectrum allocation policies traditionally enforced by spectrum regulators. This situation has recently led to the development of novel smart technologies to improve the efficiency of spectrum utilization. Relying on the principles of dynamic spectrum access and sharing and addressing all layers of the communication protocol stack, smart spectrum technologies enable the coexistence of multiple mobile wireless systems within the same spectrum band and therefore offer the potential for a smarter and more efficient exploitation of the radio spectrum in a wide range of scenarios. The research community has been working over the last years to overcome many of the technical challenges posed by the development of smart spectrum technologies. This issue compiles some of the latest advances in the field.

In response to the open call for papers, we received regular papers as well as extended versions of outstanding papers presented at the 2nd IEEE Intentional Workshop on Smart Spectrum (IWSS 2016), held in conjunction with the IEEE Wireless Communications and Networking Conference (WCNC 2016) in Doha, Qatar, on April 3, 2016. All submissions have undergone a rigorous review process and as a result six high-quality papers have been selected for publication in this special issue.

The paper titled "PSUN: An OFDM-Pulsed Radar Coexistence Technique with Application to $3.5 \mathrm{GHz}$ LTE," by $\mathrm{S}$. Kim et al. (an extended version of the paper receiving the IEEE IWSS 2016 Best Paper Award) analyzes the performance of Precoded SUbcarrier Nulling (PSUN) as a coexistence mechanism between 5G Long-Term Evolution (LTE) systems and federal military radars in the $3.5 \mathrm{GHz}$ Citizens Broadband Radio Service (CBRS) band. The pulsed radar interference can be suppressed by introducing null tones in the transmitted OFDM signal (PSUN) in addition to setting to zero (pulse-blanking) the received time-domain samples affected by pulsed interference. In this context, S. Kim et al. analyze the impact of imperfect radar pulse prediction on the performance of a PSUN OFDM system and discuss the feasibility of $5 \mathrm{G}$ applications using $3.5 \mathrm{GHz}$ LTE with PSUN.

The paper titled "CBRS Spectrum Sharing between LTEU and WiFi: A Multi-Armed Bandit Approach,” by I. Parvez et al., considers the spectral coexistence between LTE unlicensed (LTE-U) and WiFi systems in the $3.5 \mathrm{GHz}$ CBRS band. Given the contention-based channel access mechanism of WiFi systems, an unconstrained operation of LTE systems in the same band may prevent WiFi systems from accessing the spectrum. To enable a fair coexistence, LTE systems can introduce transmission gaps to allow for WiFi operation. I. Parvez et al. propose a multiarmed bandit based adaptive LTE duty cycle selection method for the dynamic optimization of these transmission gaps, which is combined with a downlink power control technique for an improved aggregate capacity and energy efficiency.

The paper titled "Licensed Shared Access System Possibilities for Public Safety," by K. Lähetkangas et al., explores the possibilities of the Licensed Shared Access (LSA) concept as an approach for spectrum sharing between public safety and commercial radio systems, taking into account the particular 
features of public safety systems, discussing the advantages and disadvantages of several spectrum sharing alternatives, and providing illustrative results on the potential benefits.

The paper titled "ETSI-Standard Reconfigurable Mobile Device for Supporting the Licensed Shared Access," by K. Kim et al., presents an implementation of a reconfigurable mobile device for LSA. The prototype implements a procedure to transfer control signals among the software entities of the device in compliance with the reference model of the ETSI standard reconfigurable architecture.

The paper titled "Spectrum Assignment Algorithm for Cognitive Machine-to-Machine Networks," by S. Rostami et al., proposes a novel aggregation-based spectrum assignment algorithm for cognitive machine-to-machine networks. $\mathrm{S}$. Rostami et al. develop a genetic algorithm taking into account practical constraints such as cochannel interference and maximum aggregation span and analyze its benefits in terms of spectrum utilization and network capacity.

The paper titled "A Survey of the DVB-T Spectrum: Opportunities for Cognitive Mobile Users," by L. CsurgaiHorváth et al., presents an experimental study of the potential opportunities offered by the terrestrial Digital Video Broadcasting (DVB-T) TV band for mobile cognitive radio applications. L. Csurgai-Horváth et al. perform a wideband spectrum survey employing a mobile measurement platform in a urban environment, where the received signal power and its statistics are analyzed in order to identify potential opportunities for mobile cognitive radio systems.

\section{Acknowledgments}

We highly appreciate the effort of all the authors in preparing and submitting their papers to this special issue as well as the dedication of the anonymous reviewers whose voluntary and invaluable work has contributed to the overall quality of this issue.

Miguel López-Benitez Janne Lehtomäki Kenta Umebayashi Fernando Casadevall 

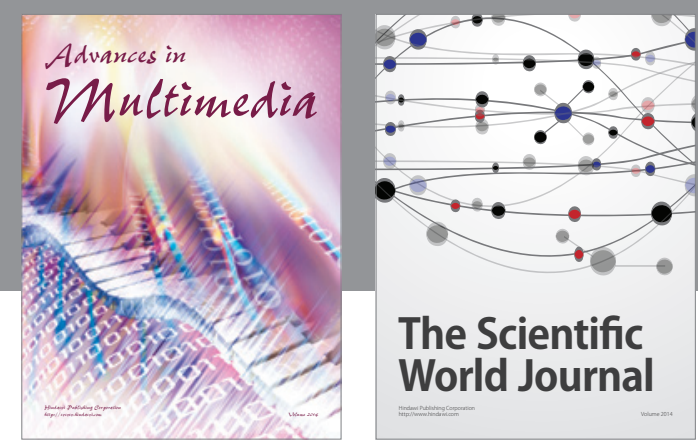

The Scientific World Journal
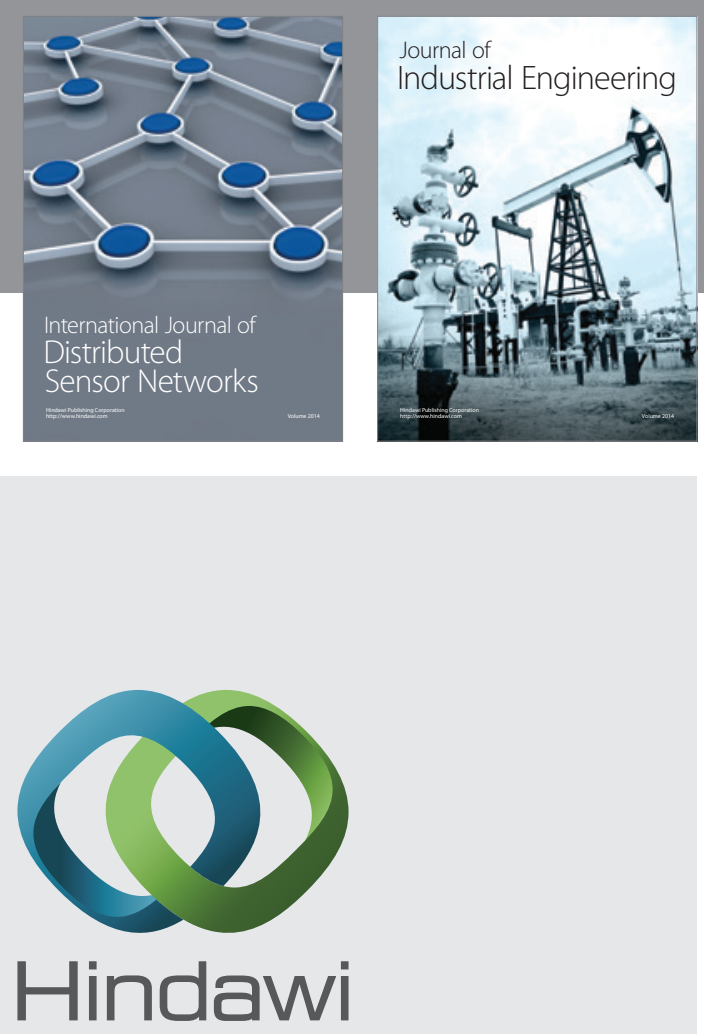

Submit your manuscripts at

http://www.hindawi.com

\section{Computer Networks} and Communications
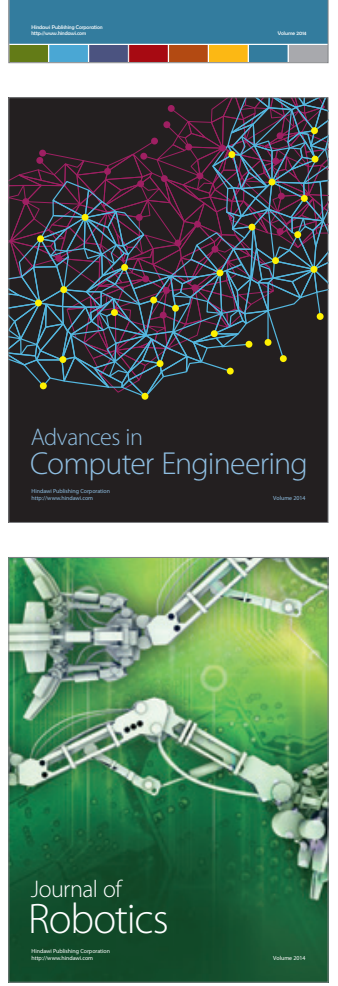
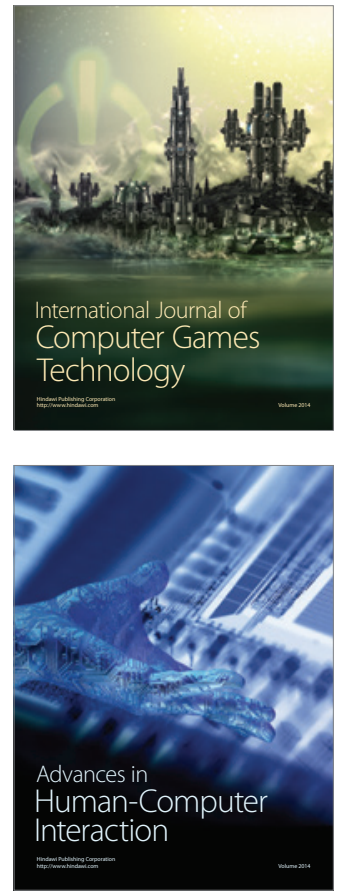
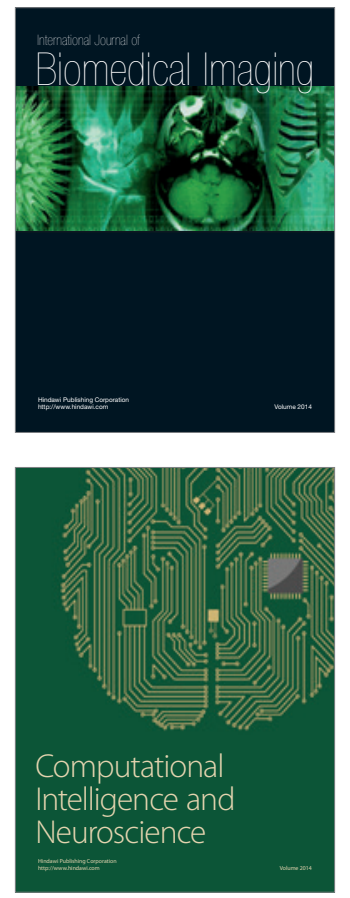
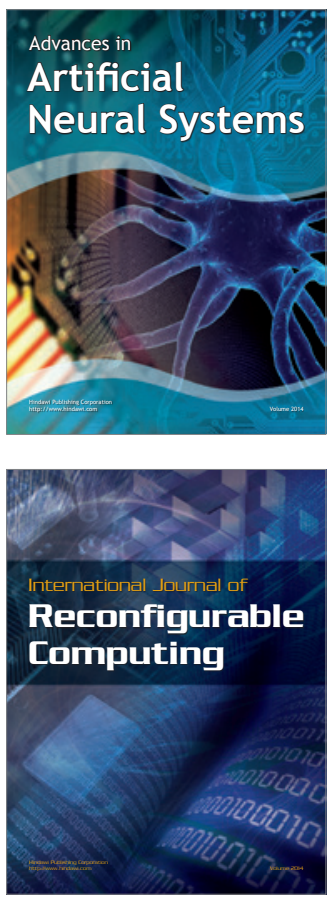
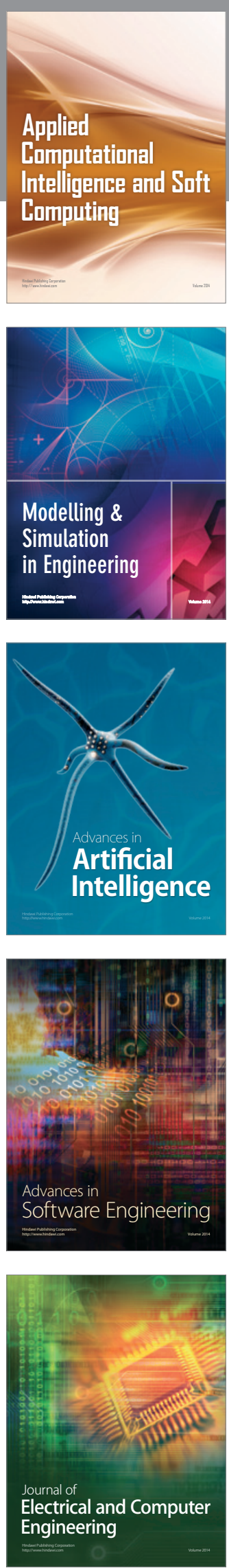